\title{
Delay-independent stability via reset loops
}

\author{
A. Baños, F. Pérez Rubio, S. Tarbouriech, L. Zaccarian
}

\section{Introduction}

An important aspect that must be taken into account in many control applications is the presence of delay in the loop, which can be the source of performance degradation or even instability (see, for example, [17], [18] and references therein).

A control architecture that received much attention in recent years is that of reset control. Reset control systems can be represented by means of different hybrid systems formulations. Many of the previous work about systems with resets has been done by using the impulsive state dependent state formulation of [10]. Alternatively, the hybrid formulation developed in $[7,8]$ has been also used for describing reset control systems, and formal stability results have been already obtained (see, e.g., $[15,14,19])$. This last framework has also been used for systems with time delays in the recent work [13], that is inspiring for the notation followed in this chapter. This formulation will be first used for stability analysis of reset control system with time delays, and with a new characterization of flow and jump sets, inspired by [6], that allows us to state a Lyapunov-Krasovskii theorem for delay-independent stability of the reset control system.

Alfonso Baños

Departamento de Informtica y Sistemas. Facultad de Informatica. Universidad de Murcia, e-mail: abanos@um.es

Félix Pérez Rubio

MTorres SAU. (31119) Spain, Software Department, e-mail: Felix.Perez@mtorres.es

Sophie Tarbouriech

CNRS, LAAS, 7 avenue du colonel Roche, F-31400 Toulouse, France and Univ de Toulouse, LAAS, F-31400 Toulouse, France, e-mail: tarbour@laas.fr

Luca Zaccarian

CNRS, LAAS, 7 avenue du colonel Roche, F-31400 Toulouse, France, Univ de Toulouse, LAAS, F-31400 Toulouse, France, and Dipartimento di Ingegneria Industriale, University of Trento, Italy e-mail: zaccarian@laas.fr 
From a historical perspective, the idea of reset compensation dates back to the seminal works of Clegg and Horowitz [5, 12], where simple reset compensators such as the Clegg integrator or the First Order Reset Element (FORE) were first proposed with the main motivation of overcoming fundamental limitations of linear time-invariant compensators in control practice. Intuitively, within this quest, since it was observed already in [5] (see also [2]) that reset compensation introduces phase lead without significantly increasing the loop gain, it seems to be appropriate to propose reset compensation for systems with time-delays due to the evident time lag introduced by the delay effect. In [1], a delay-independent stability analysis of reset control system is first performed, that has been extended to the more general delay-dependent case in [4, 2] and in [9].

In this chapter, we are interested in studying the stability of closed loops independently of the delay. In particular, our work is focused on the design of hybrid reset rules based on Lyapunov conditions and applied to time-delay continuous-time plants in order to eliminate possible instability arising from the linear interconnection. We propose a kind of hybrid controller that can guarantee delay-independent stability of the closed loop. The class of systems that we address combines two ingredients: 1) the continuous-time dynamics of a linear time-delay plant and a linear controller, enforced when the overall state belongs to a certain flow set $\mathscr{F}$ and 2) a discrete dynamics corresponding to an impulsive action performed on the controller state when the overall state belongs to a certain jump set $\mathscr{J}$ [8]. It is important to emphasize that few works are dedicated to the study of hybrid time-delay systems. Notable exceptions comprise the reset systems work in [1], [9] and the framework recently proposed in [13].

The scheme proposed in this chapter arises from adapting the hybrid augmentation paradigm recently proposed in $[16,6]$. Moreover, to prove our main result, we also formulate a Lyapunov-Krasovskii theorem that complements the results issued from [1] and [9], by using a different definition of flow and jumps sets and an additional dwell-time logic.

In the sequel we first introduce the problem under consideration in Section 2. Then we describe the proposed closed loop with resets in Section 3. Subsequently we prove suitable stability properties of the control scheme in Section 4 and finally we discuss a simulation example in Section 5.

\section{Problem data and standing assumption}

Consider the following strictly proper linear time-delay plant:

$$
\mathscr{P}:\left\{\begin{array}{l}
\dot{x}_{p}(t)=A_{p} x_{p}(t)+A_{p d} x_{p}(t-\theta)+B_{p} u_{c}(t) \\
y_{p}(t)=C_{p} x_{p}(t)
\end{array}\right.
$$

where $x_{p} \in \mathbb{R}^{n_{p}}$ is the state of the plant, $\theta \in \mathbb{R}_{\geq 0}$ is a known state delay, $y_{p} \in \mathbb{R}^{n_{y}}$ is the output available for measurement and $u_{c}$ is the control input to be used in the 
controller design. It is costumary to denote the infinite dimensional state of plant (3) as

$$
x_{p t}=\left\{x_{p}(s), s \in[t-\theta, t]\right\},
$$

and to use the shortcut notation $x_{p d}(t)=x_{p}(t-\theta)$ so that equation (3) can be written in compact from as

$$
\mathscr{P}:\left\{\begin{array}{l}
\dot{x}_{p}=A_{p} x_{p}+A_{p d} x_{p d}+B_{p} u_{p} \\
y_{p}=C_{p} x_{p}
\end{array}\right.
$$

For plant (3) we assume that a linear time invariant controller has been designed to ensure suitable closed-loop properties under certain operating conditions:

$$
\mathscr{C}:\left\{\begin{array}{l}
\dot{x}_{c}=A_{c} x_{p}+B_{c} y_{p} \\
u_{p}=C_{c} x_{c}
\end{array}\right.
$$

where $x_{c} \in \mathbb{R}^{n_{c}}$ is the state of the controller. Controller (4) has been designed for plant (3) disregarding the effect of delay, namely in such a way to stabilize the delayfree dynamics corresponding to the transition matrix $A_{p}+A_{p d}$, which corresponds to plant (3) in the special case $\theta=0$. Then the goal of this paper is to introduce suitable reset rules on the controller states that ensure recovery of closed-loop asymptotic stability for any known value of the delay $\theta$. Since we do not need the requirement that controller (4) stabilizes the plant when $\theta=0$, we don't make this as an explicit assumption.

In this work we will use Lyapunov-Krasovskii techniques to assess asymptotic stability of the closed loop for any value of the time delay $\theta$, that is, stability independent of the delay. Due to this fact, we will require the following assumption on the plant data.

Assumption 1 Given the matrices in (3) there exist two positive definite matrices $P_{p}$ and $Q$, a gain $K_{p}$ and a scalar $\varepsilon_{p}>0$ such that

$$
H e\left[\begin{array}{cc}
P_{p}\left(A_{p}+B_{p} C_{c} K_{p}\right)+Q / 2 & P_{p} A_{p d} \\
0 & -Q / 2
\end{array}\right] \leq-2 \varepsilon_{p}\left[\begin{array}{rr}
P_{p} & 0 \\
0 & Q
\end{array}\right]
$$

Assumption 1 ensures that it is possible to prestabilize plant (3) by way of the state feedback gain $u_{p}=K_{p} x_{p}$ and obtain a delay-independent stable closed loop. The following lemma clarifies that the search for parameters $P_{p}, Q$ and $K_{p}$ in (5) is equivalent to a convex (LMI eigenvalue) problem.

Lemma 1. Assumption 1 holds if and only if the following LMI in the variables $Q_{p}=Q_{p}^{T}>0, S=S^{T}>0$ and $X$ is feasible:

$$
H e\left[\begin{array}{cc}
A_{p} Q_{p}+B_{p} C_{c} X+S / 2 & A_{p d} Q_{p} \\
0 & -S / 2
\end{array}\right]<0,
$$

Moreover, whenever (6) holds, a solution to Assumption 1 is given by $P_{p}=Q_{p}^{-1}$, $Q=P_{p} S P_{p}, K_{p}=X P_{p}$ and a small enough $\varepsilon_{p}$. 
Proof. Consider equation (6) and perform the congruence transformation pre- and post-multiplying by the block-diagonal symmetric matrix $\operatorname{diag}\left(Q_{p}^{-1}, S^{-1}\right)$. Then the following equivalent relation to (6) is obtained, with the definitions in the lemma:

$$
\mathrm{He}\left[\begin{array}{cc}
P_{p}\left(A_{p}+B_{p} C_{c} K_{p}\right)+Q / 2 & P_{p} A_{p d} \\
0 & -Q / 2
\end{array}\right]<0 .
$$

Assume now that Assumption 1 holds, then obviously equation (7) holds too, which is equivalent to (6). Viceversa, if (6) (therefore (7)) holds, then due to the strict inequality in (7) there exists a small enough $\varepsilon_{p}>0$ such that (5) holds with the selections in the statement of the lemma.

Remark 1. Once the feasibility condition (6) is verified, it might be of interest to seek for the solution to (5) corresponding to maximizing $\varepsilon_{p}$ while imposing that the gain $K_{p}$ satisfies a prescribed bound $\left|K_{p}\right| \leq \kappa_{M}$, for some fixed scalar $\kappa_{M}>0$. This solution can be computed by solving the following optimization problem:

$$
\begin{aligned}
\varepsilon_{p}^{*}= & \max _{Q_{p}, X, S, \varepsilon_{p}} \varepsilon_{p} \text { subject to: } \\
& Q_{p} \geq I,\left[\begin{array}{cc}
\kappa_{M} I & X \\
X^{T} & \kappa_{M} I
\end{array}\right] \geq 0 \\
& \operatorname{He}\left[\begin{array}{cc}
A_{p} Q_{p}+B_{p} C_{c} X+S / 2 & A_{p d} Q_{p} \\
0 & -S / 2
\end{array}\right] \leq-2 \varepsilon_{p}\left[\begin{array}{cc}
Q_{p} & 0 \\
0 & S
\end{array}\right]
\end{aligned}
$$

which is a generalized eigenvalue problem (namely a quasi convex optimization problem) for which efficient numerical solution algorithms are available. The corresponding solution to (5) can then be computed as $P_{p}=Q_{p}^{-1}, Q=P_{p} S P_{p}$ and $K_{p}=X P_{p}$, just as in Lemma 1 .

\section{Hybrid closed-loop system}

In this section we design a hybrid closed-loop system whose flow dynamics corresponds to the interconnection between (3) and (4) and whose jump dynamics and jump and flow sets are constructed, based on a solution to (5) in Assumption 1, in such a way to guarantee uniform global asymptotic stability of the origin of the plant-controller state space. Note that this property is non-trivial because no assumption is made on the stability properties of the continuous-time interconnection (3), (4)

Adopting the notation in $[7,8]$, we propose the following dwell-time hybrid augmentation of the closed loop (3), (4), where for convenience of notation we denote the aggregated (and transformed) state $\xi=\left(x_{p}, x_{p d}, \delta\right):=\left(x_{p}, x_{p d}, x_{c}-K_{p} x_{p}\right)$ : 


$$
\begin{aligned}
& \left\{\begin{array}{l}
\dot{x}_{p}=A_{p} x_{p}+A_{p d} x_{p d}+B_{p} C_{c} x_{c} \\
\dot{x}_{c}=A_{c} x_{c}+B_{c} C_{p} x_{p} \\
\dot{\tau}=1-\mathrm{dz}\left(\frac{\tau}{\rho}\right)
\end{array} \quad(\xi, \tau) \in \mathscr{C} \times[0,2 \rho]\right. \\
& \left\{\begin{array}{l}
x_{p}^{+}=x_{p} \\
x_{c}^{+}=K_{p} x_{p} \\
\tau^{+}=0
\end{array} \quad(\xi, \tau) \in \mathscr{D} \times[\rho, 2 \rho],\right.
\end{aligned}
$$

where $\mathrm{dz}(\cdot)$ denotes the scalar unit deadzone function, the sets $\mathscr{C}$ and $\mathscr{D}$ are defined, based on an arbitrary positive scalar $\varepsilon<\varepsilon_{p}$, as follows:

$$
\begin{aligned}
& \mathscr{C}:=\left\{\xi: \xi^{T} \mathrm{He}\left[\begin{array}{ccc}
P_{p} A_{R}+Q / 2 & P_{p} A_{p d} & P_{p} B_{p} C_{c} \\
0 & -Q / 2 & 0 \\
0 & 0 & 0
\end{array}\right] \xi \leq-\varepsilon \xi^{T}\left[\begin{array}{ccc}
P_{p} & 0 & 0 \\
0 & Q & 0 \\
0 & 0 & I
\end{array}\right] \xi\right\} \\
& \mathscr{D}:=\left\{\xi: \xi^{T} \mathrm{He}\left[\begin{array}{ccc}
P_{p} A_{R}+Q / 2 & P_{p} A_{p d} & P_{p} B_{p} C_{c} \\
0 & -Q / 2 & 0 \\
0 & 0 & 0
\end{array}\right] \xi \geq-\varepsilon \xi^{T}\left[\begin{array}{ccc}
P_{p} & 0 & 0 \\
0 & Q & 0 \\
0 & 0 & I
\end{array}\right] \xi\right\} .
\end{aligned}
$$

In the sets $\mathscr{C}$ and $\mathscr{D}, A_{R}$ is defined as $A_{R}:=A_{p}+B_{p} C_{c} K_{p}$ and matrices $P_{p}$ and $Q$ are defined in Assumption 1.

Following [8] and inspired by the definitions in [13], we introduce the following definitions to suitably characterize solutions to hybrid system (9). In particular, the following definitions are slightly different from those in [13] because we are exploiting here the property that the memory of the time-delay system is only in the directions of the plant state, which remains unchanged across jumps.

Definition 1. A subset $E \subset \mathbb{R}_{\geq 0} \times \mathbb{Z}_{\geq 0}$ is called a compact hybrid time domain if

$$
E=\bigcup_{j=0}^{J-1}\left(\left[t_{j}, t_{j+1}\right], j\right)
$$

for some finite sequences of times $0=t_{0} \leq t_{1} \leq \cdots \leq t_{J}$, called "jump times". The set $E$ is called a hybrid time domain if for all $(T, J) \in E$, the set $E \cap([0, T],\{0,1, \ldots, J\})$ is a compact hybrid time domain.

Given a positive real $\theta>0$, a subset $E_{0} \subset \mathbb{R}_{\geq-\theta} \times \mathbb{Z}_{\leq 0}$ is called a hybrid time domain with ordinary memory $\theta$ if

$$
E_{0}=([-\theta, 0), 0) \cup E,
$$

where $E$ is a hybrid time domain.

A hybrid arc with ordinary plant memory $\theta$ is a triple consisting of a domain $\operatorname{dom} \phi$ that is a hybrid time domain with ordinary memory $\theta$, a continuous function $\phi_{p 0}(s), s \in[-\theta, 0]$ representing the (infinite dimensional) initial condition of the system in the plant state direction and a function $\phi: \operatorname{dom}_{\geq 0} \phi \rightarrow \mathbb{R}^{n_{p} \times n_{c}}$, where $\operatorname{dom}_{\geq 0} \phi:=\operatorname{dom} \phi \cap\left(\mathbb{R}_{\geq 0} \times \mathbb{Z}_{\geq 0}\right)$, such that $\phi(\cdot, j)$ is locally absolutely continuous on $I^{j}=\left\{t:(t, j) \in \mathrm{dom}_{\geq 0} \phi\right\}$. 
Similar to [13, Def. 4], but for the special case where the memory of the timedelay system is only in the ordinary time direction, given any hybrid $\operatorname{arc} \phi=\left(\phi_{p}, \phi_{c}\right)$ with ordinary plant memory $\theta$, for each $(t, j) \in \operatorname{dom}_{\geq 0} \phi$, we define the operator $\mu_{[t, j]} \phi_{p}(s)$ that maps the hybrid arc $\phi$ into an ordinary memory arc of length $\theta$ (this is a function of ordinary time only). In particular, $\operatorname{dom} \mu_{[t, j]} \phi_{p}=[-\theta, 0]$ and for each $s \in[-\theta, 0]$ we have $\mu_{[t, j]} \phi_{p}(s)=\phi_{p}(t+s, i)$ for some $i \in \mathbb{Z}_{\geq 0}$ such that $(t-s, i) \in \operatorname{dom} \phi$. Note that such an $i$ exists because the hybrid arc $\phi$ has ordinary plant memory $\theta$ by assumption. Note also that the definition above has no ambiguity because of the special structure in (9) where the $p$ component of the solution remains constant across jumps. Indeed, if there exist multiple values $i_{1}, i_{2}, \in \mathbb{Z}_{\geq 0}$ such that $\left(t-s, i_{1}\right),\left(t-s, i_{2}\right) \in \operatorname{dom} \phi$, then we have $\mu_{[t, j]} \phi_{p}(s)=\phi_{p}\left(t+s, i_{1}\right)=\phi_{p}(t+$ $\left.s, i_{2}\right)$. Indeed, projecting the memory of the time-delay system only on the ordinary time domain axis (by way of the operator $\mu_{[t, j]}$ ) greatly simplifies the forthcoming derivations.

Based on the above characterization, we can formulate a class of hybrid timedelay systems that generalizes the peculiar structure of (9) and that can be written in compact form as follows:

$$
\begin{aligned}
& {\left[\begin{array}{c}
\dot{x}_{p} \\
\dot{x}_{c} \\
\dot{\tau}
\end{array}\right]=\left[\begin{array}{c}
f_{p}\left(x_{p}, x_{c}, \mu x_{p}\right) \\
f_{c}\left(x_{p}, x_{c}, \mu x_{p}\right) \\
1-\mathrm{dz}\left(\frac{\tau}{\rho}\right)
\end{array}\right],\left[\begin{array}{c}
x_{p} \\
x_{c} \\
\mu_{p} \\
\tau
\end{array}\right] \in \widetilde{\mathscr{C}} \times[0,2 \rho], \quad\left(x_{p}(0,0), x_{c}(0,0)\right) \in \mathbb{R}^{n_{p} \times n_{c}}} \\
& {\left[\begin{array}{c}
x_{p}^{+} \\
x_{c}^{+} \\
\tau^{+}
\end{array}\right]=\left[\begin{array}{c}
x_{p} \\
g_{c}\left(x_{p}, x_{c}, \mu x_{p}\right) \\
0
\end{array}\right],\left[\begin{array}{c}
x_{p} \\
x_{c} \\
\mu_{p} \\
\tau
\end{array}\right] \in \widetilde{\mathscr{D}} \times[\rho, 2 \rho],}
\end{aligned}
$$

where $\mathscr{C}^{0}(\mathscr{T})$ denotes the set of continuous functions whose domain is $\mathscr{T} \subset \mathbb{R}, f_{p}$, $f_{c}$ and $g_{c}$ are suitable linear functionals that, for system (9), correspond to:

$$
\begin{aligned}
& f_{p}\left(x_{p}, x_{c}, \mu x_{p}\right)=A_{p} x_{p}+A_{p d} \mu x_{p}(-\theta)+B_{p} C_{c} x_{c} \\
& f_{c}\left(x_{p}, x_{c}, \mu x_{p}\right)=A_{c} x_{c}+B_{c} C_{p} x_{p} \\
& g_{c}\left(x_{p}, x_{c}, \mu x_{p}\right)=K_{p} x_{p} .
\end{aligned}
$$

Moreover, the flow and jump sets $\widetilde{\mathscr{C}}$ and $\widetilde{\mathscr{D}}$ are suitable infinite dimensional flow and jump sets.

Based on Definition 1, a solution to hybrid time-delay system (10) is a hybrid arc with ordinary plant memory $\theta$ satisfying the flow and jump constraints imposed by the hybrid dynamics. A precise formulation of this can be obtained by following the same paradigm as that used in [13, Def. 6].

Remark 2. The extension of the formalism in $[7,8]$ to the time-delay framework is currently underdeveloped (a notable exception being the recent paper [13]). Therefore, not much can be stated about existence of solutions or even nominal or robust well posedness of solutions to these hybrid systems in the sense of [8, Ch. 6]. Results on well posedness of a particular class of hybrid systems is given in [13], however system (10) goes beyond this class because in [13] it is assumed that the flow and 
jump sets are subsets of the Euclidean spaces where $x_{p}$ and $x_{c}$ take values. Here we have a new direction in the jump and flow sets depending on the (infinite dimensional) memory of the plant state $x_{p}$ and it is not evident how to inherit the results of [13]. However, at least from the existence viewpoint, the architecture (10) can still inherit useful properties from the classical results in [11] and from the results in [8, Ch. 6], as long as the initial condition $\mu_{[0,0]} x_{p}$ of the plant is continuous.

Remark 3. Note that differently from [6], the dwell-time logic is implemented in (9) by forcing solutions not to jump unless $\tau \geq \rho$, while also not allowing them to flow if their $\xi$ component does not belong to the set $\mathscr{C}$. Because of this fact, dwell time is artificially enforced on solutions by possibly terminating defective solutions that would jump too often. The natural question that arises is then whether system (9) admits complete solutions for all initial conditions starting either in the jump or in the flow set. This question is not addressed here, where we simply limit ourselves to observing that the example treated in Sect. 5 exhibits complete solutions. We regard tackling this important aspect as future work.

\section{Stability properties of the reset control scheme}

\subsection{A Lyapunov-Krasovskii theorem}

We state in this section a peculiar version of a Lyapunov-Krasovskii theorem for system (10). Alternative instances of this type of result have appeared in the recent literature. For example see [1, Prop. 3.1] or [9, Prop. 1]. We state here a different formulation of the result, due to the special definitions and hybrid framework used in the previous section.

Since we are not interested in the evolution of the timer $\tau$ within the compact set $[0,2 \rho]$ where it is confined, we will characterize stability properties of the following compact attractor for dynamics (10):

$$
\mathscr{A}:=\{0\} \times[0,2 \rho] \subset \mathbb{R}^{n_{p}+n_{c}} \times \mathbb{R} .
$$

In particular, following standard derivations in the time-delay continuous-time systems framework, given any solution $\phi=\left(\phi_{x}, \phi_{\tau}\right)=\left(\phi_{p}, \phi_{c}, \phi_{\tau}\right)$ to (10), we introduce the following notion of distance from the set $\mathscr{A}$ in (11) for each $(t, j) \in \operatorname{dom}_{\geq 0} \phi$ :

$$
\|\phi(t, j)\|_{\mu}=\max \left\{\left|\phi_{x}(t, j)\right|, \max _{s \in[-\theta, 0]}\left|\mu_{[t, j]} \phi_{p}(s)\right|\right\},
$$

where $|\cdot|$ denotes the Euclidean norm.

Since we are dealing with a special class of hybrid time-delay systems, we clarify the meaning of stability in the following definition, which is inspired by [13] and [8, $\S 3.1]$. 
Definition 2. The compact set $\mathscr{A}$ in (11) is

1. Globally stable (GS) for (10) if there exists a class $\mathscr{K}_{\infty}$ function $\alpha$ such that any solution $\phi=\left(\phi_{x}, \phi_{\tau}\right)=\left(\phi_{p}, \phi_{c}, \phi_{\tau}\right)$ to (10) satisfies $\left|\phi_{x}(t, j)\right| \leq \alpha\left(\|\phi(0,0)\|_{\mu}\right)$ for all $(t, j) \in \operatorname{dom}_{>0} \phi$.

2. Uniformly globally attractive (UGA) for (10) if for each pair $r, \varepsilon$, there exists $T(r, \mathcal{E})$ such that any solution $\phi$ satisfies:

$\|\phi(0,0)\|_{\mu} \leq r \Rightarrow\left|\phi_{x}(t, j)\right| \leq \varepsilon, \forall(t, j) \in \operatorname{dom}_{\geq 0} \phi$ such that $t+j \geq T(r, \varepsilon)$.

3. Uniformly globally asymptotically stable for (10) if it is GS and UGA.

Based on Definition 2 we can state a Lyapunov-Krasovskii result that only requires the following mild uniform boundedness assumption on the functions appearing in the flow map of (10). This assumption is trivially satisfied by the linear flow dynamics in (9).

Assumption 2 There exists a class $\mathscr{K}_{\infty}$ function $\alpha_{M}$ such that for each $r \geq 0$ (where we use $\left.x=\left(x_{p}, x_{c}\right)\right)$,

$$
\sup _{\left(x, \mu x_{p}\right) \in \mathscr{C} \text { s.t. }\|x\| \mu \leq r}\left[\begin{array}{l}
f_{p}\left(x, \mu x_{p}\right) \\
f_{c}\left(x, \mu x_{p}\right)
\end{array}\right] \leq \alpha_{M}(r)
$$

Proposition 1. Under Assumption 2, if there exist a function $V$, two class $\mathscr{K}_{\infty}$ functions $\alpha_{1}, \alpha_{2}$ and a positive definite function $\sigma$ satisfying

$$
\begin{array}{lr}
\alpha_{1}(|x|) \leq V\left((x, \tau), \mu x_{p}\right) \leq \alpha_{2}\left(\|(x, \tau)\|_{\mu}\right), & \forall\left(x, \mu x_{p}, \tau\right) \\
\dot{V}\left((x, \tau), \mu x_{p}\right) \leq-\rho(|x|), & \forall\left(x, \mu x_{p}, \tau\right) \in \widetilde{\mathscr{C}} \times[0,2 \rho], \\
V\left(\left(x^{+}, \tau^{+}\right), \mu x_{p}\right)-V\left((x, \tau), \mu x_{p}\right) \leq 0, & \forall\left(x, \mu x_{p}, \tau\right) \in \widetilde{\mathscr{D}} \times[\rho, 2 \rho],
\end{array}
$$

then the compact attractor $\mathscr{A}$ in (11) is uniformly globally asymptotically stable for (10).

Proof. The proof is omitted due to space constraints but can be found in [3].

\subsection{Main stability result}

We state next our main stability result for the hybrid dynamics (9). To properly state the stability result,

Theorem 1. Consider a plant (3) satisfying Assumption 1 and a controller (4). Then for the dwell-time hybrid time-delay dynamics (9) there exist a functional $V$ and class $\mathscr{K}_{\infty}$ functions $\alpha_{1}, \alpha_{2}$ and $\sigma$ satisfying (14)-(16). Namely, the set $\mathscr{A}$ in (11) is globally asymptotically stable. 
Proof. Using $P_{p}$ and $Q$ of Assumption 1, consider the following Lyapunov-Krasovskii functional for the plant state directions:

$$
V_{p}\left(x_{p}, \mu x_{p}\right)=x_{p}^{T} P_{p} x_{p}+\int_{-\theta}^{0} \mu x_{p}^{T}(s) Q \mu x_{p}(s) d s .
$$

The derivative of $V_{p}$ along the flow dynamics of (9) is (for notational compactness, we use $x_{p d}:=\mu x_{p}(-\theta)$ in the rest of the proof):

$$
\begin{aligned}
& \dot{V}_{p}=2 x_{p}^{T} P_{p}\left[A_{p} x_{p}+B_{p} C_{c}\left(K_{p} x_{p}+x_{c}-K_{p} x_{p}\right)+A_{p d} x_{p d}\right]+x_{p}^{T} Q x_{p}-x_{p d}^{T} Q x_{p d} \\
& =2 x_{p}^{T} P_{p}\left[(\underbrace{A_{p}+B_{p} C_{c} K_{p}}_{A_{R}}) x_{p}+A_{p d} x_{p d}+B_{p} C_{c}(\underbrace{x_{c}-K_{p} x_{p}}_{\delta})\right]+x_{p}^{T} Q x_{p}-x_{p d}^{T} Q x_{p d} \\
& =\left[\begin{array}{c}
x_{p} \\
x_{p d} \\
\delta
\end{array}\right]^{T} \mathrm{He}\left[\begin{array}{ccc}
P_{p} A_{R}+Q / 2 & P_{p} A_{p d} & P_{p} B_{p} C_{c} \\
0 & -Q / 2 & 0 \\
0 & 0 & 0
\end{array}\right]\left[\begin{array}{c}
x_{p} \\
x_{p d} \\
\delta
\end{array}\right]
\end{aligned}
$$

Then, from the definition (9b) of the flow set $\mathscr{C}$ we have that

$$
\dot{V}_{p} \leq-\varepsilon\left(\left|x_{p}\right|_{P_{p}}^{2}+\left|x_{p d}\right|_{Q}^{2}+|\delta|^{2}\right), \quad \forall\left(x_{p}, x_{p d}, \delta\right) \in \mathscr{C}
$$

where $|y|_{W}=\sqrt{y^{T} W y}$.

Now consider the following Lyapunov-Krasovskii functional for the overall system:

$$
V=V_{p}\left(x_{p}, \mu x_{p}\right)+\lambda \delta^{2},
$$

where $\lambda$ is a positive scalar selected later. We show next that this function satisfies (14)-(16), so that the result follows from Proposition 1.

Proof of (14). The lower bound on the left comes from

$$
x_{p}^{T} P_{p} x_{p}+\lambda \delta^{2}=\left[\begin{array}{c}
x_{p} \\
x_{c}
\end{array}\right]^{T} \underbrace{\left[\begin{array}{cc}
P_{p}+\lambda K_{p}^{T} K_{p} & -\lambda K_{p} \\
-\lambda K_{p}^{T} & \lambda I
\end{array}\right]}_{\Sigma}\left[\begin{array}{l}
x_{p} \\
x_{c}
\end{array}\right] \geq \sigma_{m}(\Sigma)\left|\left[\begin{array}{l}
x_{p} \\
x_{c}
\end{array}\right]\right|^{2}=: \alpha_{1}\left(\left|\left[\begin{array}{l}
x_{p} \\
x_{c}
\end{array}\right]\right|\right),
$$

where $\sigma_{m}(\Sigma)>0$ because $\Sigma$ is positive definite.

For the upper bound we get:

$$
\begin{aligned}
V\left(x, \mu x_{p}\right) & \leq\left[\begin{array}{l}
x_{p} \\
x_{c}
\end{array}\right]^{T} \Sigma\left[\begin{array}{l}
x_{p} \\
x_{c}
\end{array}\right]+\int_{-\theta}^{0} \sigma_{M}(Q)\left|\mu x_{p}(s)\right|^{2} d s \\
& \leq \sigma_{M}(\Sigma)\left|\left[\begin{array}{l}
x_{p} \\
x_{c}
\end{array}\right]\right|^{2}+\theta \sigma_{M}(Q) \max _{s \in[-\theta, 0]}\left|\mu x_{p}(s)\right|^{2} \\
& \leq 2 \max \left\{\sigma_{M}(\Sigma), \theta \sigma_{M}(Q)\right\}\|(x, \tau)\|_{\mu}^{2}=: \alpha_{2}\left(\|(x, \tau)\|_{\mu}\right) .
\end{aligned}
$$


where $\sigma_{M}(\cdot)$ and $\sigma_{m}(\cdot)$ denote, respectively, the maximum and minimum singular values of their arguments.

Proof of (15). First, we may easily compute:

$$
\dot{V} \leq \dot{V}_{p}+\lambda \delta\left((\underbrace{A_{c}-K_{p} B_{p} C_{c}}_{A_{1}}) x_{c}+(\underbrace{B_{c} C_{p}-K_{p} A_{p}}_{A_{2}}) x_{p}+(\underbrace{-K_{p} A_{p d}}_{A_{3}}) x_{p d}\right) .
$$

Since $x_{c}=\delta+K_{p} x_{p}$, then using (19), equation (21) can be rewritten as

$$
\begin{aligned}
\dot{V} & \leq-\varepsilon\left|x_{p}\right|_{P_{p}}^{2}-\varepsilon\left|x_{p d}\right|_{Q}^{2}-\varepsilon|\delta|^{2}+\lambda \delta\left(A_{1} \delta+\left(A_{1} K_{p}+A_{2}\right) x_{p}+A_{3} x_{p d}\right) \\
& \leq-\varepsilon \underline{c}\left|x_{p}\right|^{2}-\varepsilon \underline{c}\left|x_{p d}\right|^{2}-\varepsilon|\delta|^{2}+\lambda \bar{c}\left(|\delta|^{2}+|\delta|\left|x_{p}\right|+|\delta|\left|x_{p d}\right|\right)
\end{aligned}
$$

where $\bar{c}=\max \left\{\sigma_{M}\left(A_{1}\right), \sigma_{M}\left(A_{1} K_{p}+A_{2}\right), \sigma_{M}\left(A_{3}\right)\right\}$ and $\underline{c}=\min \left\{\sigma_{m}\left(P_{p}\right), \sigma_{m}(Q)\right\}$.

Finally, completing squares and choosing $\lambda=\varepsilon \min \left\{\frac{\bar{c}}{\bar{c}}, \frac{1}{4 \bar{c}}\right\}$, we get

$$
\begin{aligned}
\dot{V} & \leq-\left(\varepsilon \underline{c}-\frac{\lambda \bar{c}}{2}\right)\left|x_{p}\right|^{2}-\left(-\varepsilon \underline{c}+\frac{\lambda \bar{c}}{2}\right)\left|x_{p d}\right|^{2}-(\varepsilon-2 \lambda \bar{c})|\delta|^{2} \\
& \leq-\frac{\varepsilon}{2}\left|\left[\begin{array}{cr}
I & 0 \\
-K_{p} & I
\end{array}\right]\right|\left|\left[\begin{array}{c}
x_{p} \\
x_{c}
\end{array}\right]\right|^{2}=:-\sigma\left(\left|\left(x_{p}, x_{c}\right)\right|^{2}\right),
\end{aligned}
$$

which implies the flow condition (15).

Proof of (16). Simply observe that $x_{p}$ remains constant across jumps, therefore

$$
V\left(x^{+}, \mu x_{p}\right)-V\left(x, \mu x_{p}\right)=\lambda\left(\left(\delta^{+}\right)^{2}-\delta^{2}\right)=-\delta^{2} \leq 0,
$$

where we used the fact that $\delta$ is reset to zero at each jump.

\section{Simulation example}

Consider the following entries for the matrices in (3):

$$
A_{p}=\left[\begin{array}{cc}
-2 & 0 \\
0 & -0.9
\end{array}\right], A_{p d}=\left[\begin{array}{cc}
-1 & 0 \\
-1 & -1
\end{array}\right], B_{p}=\left[\begin{array}{l}
1 \\
1
\end{array}\right], C_{p}=\left[\begin{array}{ll}
1 & 1
\end{array}\right]
$$

and the selection for controller (4):

$$
A_{c}=\left[\begin{array}{cc}
-1 & 0 \\
1 & 0
\end{array}\right], B_{c}=\left[\begin{array}{l}
1 \\
0
\end{array}\right], C_{c}=[0-1]
$$

which involves an integral action possibly to deal with constant references or disturbances. When interconnecting plant and controller, we obtain a continuous-time dynamics as in the upper equation of (9a) with 


$$
\begin{gathered}
A_{p}=\left[\begin{array}{cc}
-2 & 0 \\
0 & -0.9
\end{array}\right], A_{p d}=\left[\begin{array}{cc}
-1 & 0 \\
-1 & -1
\end{array}\right], B_{p} C_{c}=\left[\begin{array}{ll}
0 & -1 \\
0 & -1
\end{array}\right], \\
A_{c}=\left[\begin{array}{cc}
-1 & 0 \\
1 & 0
\end{array}\right], B_{c} C_{p}=\left[\begin{array}{ll}
1 & 1 \\
0 & 0
\end{array}\right] .
\end{gathered}
$$

Such a continuous-time dynamics is exponentially stable if $\theta=0$ (case with no delay) but for larger delays, beyond the critical value $\theta_{M}=1.6$, the (linear) continuous-time closed loop becomes unstable.

Plant (3), (22) satisfies Assumption 1, therefore we may follow our hybrid construction to obtain GAS of the origin for any value of the delay, while preserving the continuous-time dynamics induced by (4), (24). To this aim, we follow the design paradigm in Remark 1 and compute a trade-off curve between the bound $\kappa_{M}>0$ and the decrease rate $\varepsilon_{p}^{*}$.

Fig. 1 shows the optimal values of $\varepsilon_{p}^{*}$ as a function of the bound $\kappa_{M}$ on $\left|K_{p}\right|$. Note that if $\kappa_{M}$ is too small, then $\varepsilon_{p}^{*}$ is negative and the design cannot be performed. Clearly, the curve is nondecreasing as increasing $\kappa_{M}$ one enlarges the feasible set.

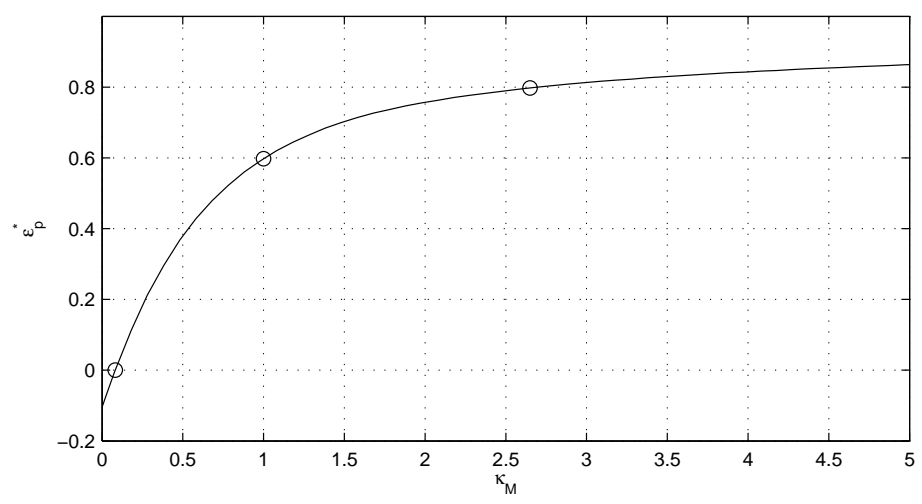

\begin{tabular}{|c|c|c|c|}
\hline & $\kappa_{m}$ & $\varepsilon_{p}^{*}$ & $K_{p}$ \\
\hline Case 1 & 0.081 & $3.6 \cdot 10^{-4}$ & {$\left[\begin{array}{cc}0.0148 & -0.0001 \\
-0.0591 & -0.0542\end{array}\right]$} \\
\hline Case 2 & 1 & 0.5976 & {$\left[\begin{array}{cc}0.4501 & -0.1268 \\
-0.6102 & -0.7634\end{array}\right]$} \\
\hline Case 3 & 2.65 & 0.7977 & {$\left[\begin{array}{rr}1.3365 & -1.2358 \\
-1.3268 & -2.2350\end{array}\right]$} \\
\hline
\end{tabular}

Fig. 1 The optimal value $\varepsilon_{p}^{*}$ in (8) as a function of $\kappa_{M}$.

Table 1 The three cases addressed in the simulation example. 
Table 1 shows the optimal values corresponding to the three circles reported in Fig. 1. The first value is just after the stability limit and the two other ones correspond to different trade-offs between $\varepsilon_{p}^{*}$ and $\kappa_{M}$. For these three cases we run a time simulation selecting $\theta=2$.
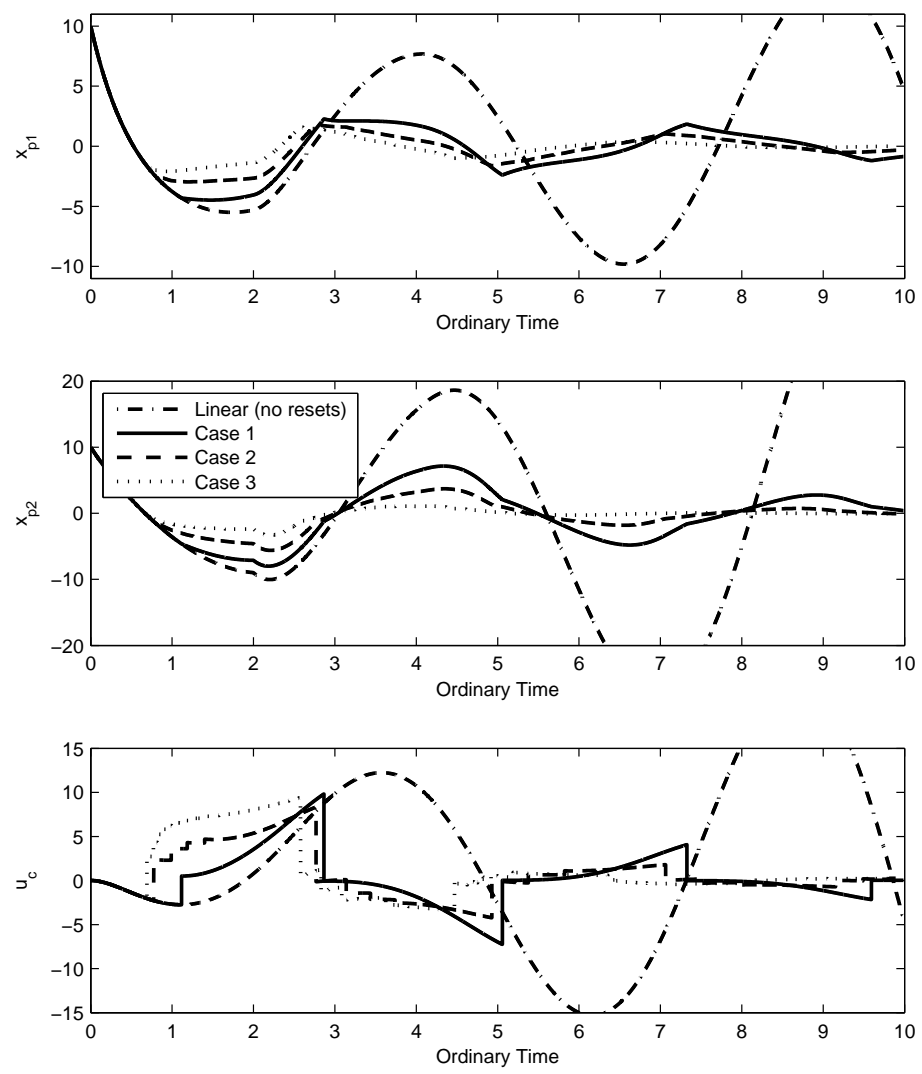

Fig. 2 States and input of the plant for the simulation test.

The simulation results for cases 1, 2, and 3, respectively, are shown in Figs. 2 and 3 using solid, dashed, and dotted curves, respectively. In the two figures, we also show the linear response (no resets), which is diverging (dash-dotted curves) because $2>\theta_{M} \approx 1$.6. For the simulations, we selected $\rho=0.01 s$ that turns out to be well below the time elapsed between any pair of consecutive resets. Therefore, with reference to Remark 3, the dwell-time logic does not prematurely terminate our solutions.

The simulation results confirm the faster convergence rate (larger $\varepsilon_{p}^{*}$ ) envisioned for larger values of $\kappa_{M}$. Quite interestingly, this faster convergence is obtained by resetting earlier, rather than using a larger control input. Indeed, for larger values of 

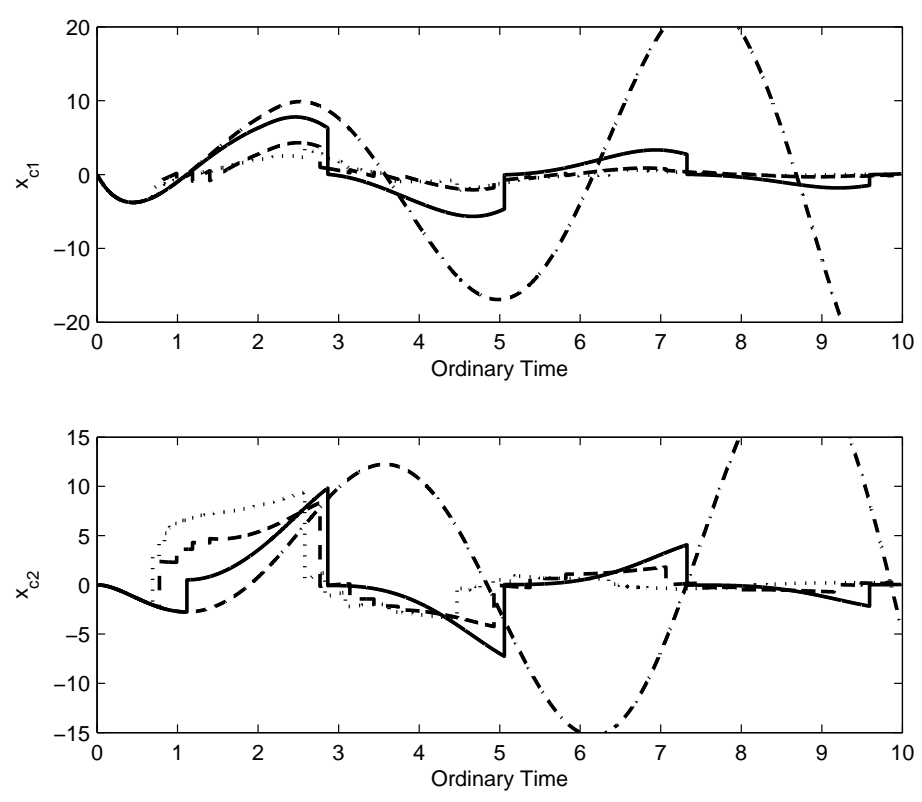

Fig. 3 States of the controller for the simulation test.

$\kappa_{M}$, we observe a reduced amplitude of the control input $u_{c}$ (see the bottom plot in Fig. 2).

Acknowledgements This work was supported by ANR project LimICoS contract number 12 BS03 005 01, by HYCON2 Network of Excellence "Highly- Complex and Networked Control Systems", grant agreement 257462 and by MICINN under the project DPI 2007-66455-C02-01.

\section{References}

1. A. Baños and A. Barreiro. Delay-independent stability of reset systems. IEEE Transactions on Automatic Control, 54(2):341-346, 2009.

2. A. Baños and A. Barreiro. Reset Control Systems, AIC Series, Springer, London, 2012

3. A. Baños, F. Pérez Rubio, S. Tarbouriech, and L. Zaccarian Delay-independent stability via reset loops LAAS-CNRS technical report, 2013.

4. A. Barreiro and A. Baños. Delay-dependent stability of reset systems, Automatica, 46(1):216221,2010

5. J.C. Clegg. A nonlinear integrator for servomechnisms, Transactions A.I.E.E.m, Part II, 77:4142, 1958.

6. F. Fichera, C. Prieur, S. Tarbouriech, and L. Zaccarian. Using Luenberger observers and dwelltime logic for feedback hybrid loops in continuous-time control systems. International Journal of Robust and Nonlinear Control, 23:1065-1086, 2013.

7. R. Goebel, R. Sanfelice, and A.R. Teel. Hybrid dynamical systems. Control Systems Magazine, IEEE, 29(2):28-93, April 2009. 
8. R. Goebel, R.G. Sanfelice, and A.R. Teel. Hybrid Dynamical Systems: modeling, stability, and robustness. Princeton University Press, 2012.

9. Y. Guo and L. Xie. Quadratic stability of reset control systems with delays. In World congress on intelligent control and automation, pages 2268-2273, July 2012.

10. W.M. Haddad, V. Chellaboina, and S.G. Nersesov. Impulsive and hybrid dynamical systems: stability, dissipativity, and control, Princeton University Press, 2006.

11. J.K. Hale and S.M.V. Lunel. Introduction to functional differential equations, volume 99 of Applied Mathematical Sciences. Springer, 1993.

12. I.M. Horowitz and P. Rosenbaum. Nonlinear design for cost of feedback reduction in systems with large parameter uncertainty, International Journal of Control, 24(6):977-1001, 1975.

13. J. Liu and A.R. Teel. Generalized solutions to hybrid systems with delays. In Conference on Decision and Control, pages 6169-6174, Maui (HI), USA, December 2012.

14. D. Nešić, A.R. Teel, and L. Zaccarian. Stability and performance of SISO control systems with First Order Reset Elements. IEEE Transactions on Automatic Control, 56(11):2567-2582, 2011.

15. D. Nesic, L. Zaccarian, and A.R. Teel. Stability properties of reset systems", Automatica, 44:2019-2026, 2008.

16. C. Prieur, S. Tarbouriech, and L. Zaccarian. Lyapunov-based hybrid loops for stability and performance of continuous-time control systems. Automatica, 49(2):577-584, 2013.

17. R. Sipahi, S. Niculescu, C. T. Abadallah, W. Michiels, and K. Gu. Stability and stabilization of systems with time delay, IEEE Control Systems Magazine, 31(1):38-65, 2011.

18. R. Sipahi, T. Vyhlídal, S. Niculescu, and P. Pierdomenico (Eds). Time delay systems: method, applications and new trends, Springer, 2012.

19. L. Zaccarian, D. Nešić, and A.R. Teel. Analytical and numerical Lyapunov functions for SISO linear control systems with First Order Reset Elements. Int. J. Robust and Nonlinear Control, 21(10):1134-1158, 2011. 